\title{
Study of Solar Gamma Rays basing on Geant4 code
}

\author{
Baosheng Gao*t \\ University of Liaoning, Shenyang 110036, China \\ University of Nankai, Tianjin 300071, China \\ Institute of High Energy Physics, Chinese Academy of Sciences, Beijing, 100049, China \\ E-mail: gaobseihep.ac.cn

\section{Songzhan Chen} \\ Institute of High Energy Physics, Chinese Academy of Sciences, Beijing, 100049, China
}

\author{
Chunxu Yu \\ University of Nankai, Tianjin 300071, China
}

\section{Kuiyong Liu}

University of Liaoning, Shenyang 110036, China

\author{
Huihai He \\ Institute of High Energy Physics, Chinese Academy of Sciences, Beijing, 100049, China
}

\section{Zhe Li}

Institute of High Energy Physics, Chinese Academy of Sciences, Beijing, 100049, China

\begin{abstract}
The solar gamma-ray are mainly produced by the hadronic interactions of cosmic rays with the solar atmosphere. Some theoretical works have been done to estimate the flux distribution of solar gamma-ray by Monte Carlo simulation based. In this work, we will estimate the solar gamma-ray emission based on Geant 4 code. An approximate structure of the Sun and its atmosphere are constructed in the code. The cosmic rays are generated at $600 \mathrm{~km}$ above the solar surface. The gamma-ray are traced until they are absorbed or escape from the solar atmosphere. Finally, the flux and spectrum for observers on Earth are presented. Some comparison between our result and others are also discussed.
\end{abstract}

35th International Cosmic Ray Conference - ICRC2017

10-20 July, 2017

Bexco, Busan, Korea

\footnotetext{
*Speaker.

${ }^{\dagger}$ This work is supported in China by N SFC N o.11575203
} 


\section{Introduction}

Sun is a significant gamma-ray source, and the mechanism of gamma-ray generation is various. The well known gamma-ray are generated by the interaction of flare-accelerated particle in the solar atmosphere. Until now, some of these kind of gamma-ray have been detected by the Fermi Large Area Telescope (LAT) between $100 \mathrm{MeV}$ [四] and $4 \mathrm{GeV}$ []]. Another possible mechanism the gamma-ray is the self-annihilation of dark matter, but there is not find enough experimental evidence[[3] [四], we don't discussed in this paper. The most significant astrophysical mechanism for steady solar gamma-ray production is the interactions of cosmic rays with solar atmosphere,

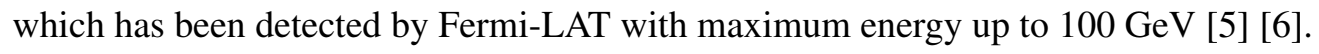

Cosmic rays ,entering the atmosphere interact with atmosphere, produce the solar gamma-ray, which was firstly proposed by Dolan et al.(1965) [四], the energy range we known was from 20 $\mathrm{keV}$ to $10 \mathrm{MeV}[[]]$, a more detailed theoretical model for gamma-ray from the interaction between cosmic ray and the solar atmosphere is presented by Seckel et al.(1991)[Q]. The predicted gammaray flux at energy from $10 \mathrm{MeV}$ to $10 \mathrm{GeV}$ has a large uncertainty, because cosmic rays are affected by magnetic field near the Sun.

The predicted gamma-ray was firstly detected by Energetic gamma-ray Experiment Telescope (EGRET) (Orlando et al. 2008)[प]]. The measured flux in the low energy range, which from 100 $\mathrm{MeV}$ to $2 \mathrm{GeV}$, was within the range of theoretical prospection. Recently, the Fermi collaboration use the data of Fermi-LAT 6 years to analyze and found that a significant time variation that anti-correlate with solar activity, which inferred that the solar magnetic field would play an very important role, which is also our the focus of the next step work. The solar gamma-ray measured in the $\mathrm{GeV}$ range with Fermi data are high compared with the previous experiments and higher extrapolation suggests that HAWC and LHAASO may detect gamma-ray in the TeV range. These experiments will open a possibility to us for higher energy, but due to the direct measurements are limited and the traditional theoretical calculations are not appropriate for the nearly hundreds of scattering. So in order to offer these experimental presumptive observation better, We use Geant4, which is a software developed by European Organization for Nuclear Research be aimed at simulating reactions between particles, to simulate the interaction of the cosmic rays with the sun's atmosphere.

This paper makes steps towards a comprehensive understanding of solar gamma-ray. In Sec.II, we introduce the calculation based on Geant 4 code In Sec.III, we introduce the method of analysis and show some important data. In Sec.IV, We show some results and compare the results with other experiments. In Sec.V, we present our conclusions and the outlook for further works.

\section{Simulation based On Geant4 Code}

Solar gamma-ray detector structure is mainly consists of two parts, the first part is the solar atmosphere structure. In this section we introduce the process of cosmic ray entering into the atmosphere of Sun and producing the gamma-ray. The cosmic ray particles enter into the solar atmosphere, only very few of them intact with solar atmosphere, at the same time the gamma-ray and other particles are being produced shown in the fig.1. We adjust the size of the solar atmosphere and the structure of the solar photosphere in order to show you the process more clearly. In this 


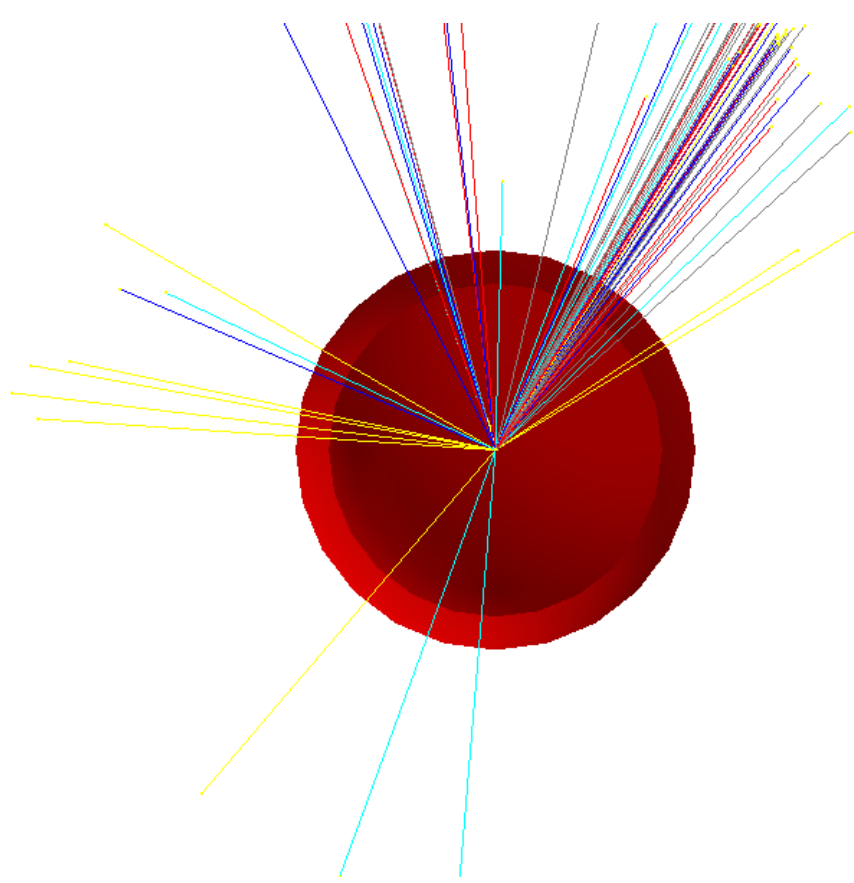

Figure 1: As we shown, deep red area represent photosphere and light red represent solar atmosphere, different color line represent different particles, the gray line represent proton, the green line represent gamma-ray, other lines represent other particles. In this figure most cosmic rays enter into the solar atmosphere without interacting. Very few of them interact with solar atmosphere and produce the solar gamma-ray and other particles, only generate gamma-ray information recording on the edge of the atmosphere.

section, there are two problems should be concerned; the first one is that the density of the solar atmosphere continuity with height; Secondly, we need set the energy threshold for saving time. When the energy of particles below the energy threshold, the interaction will be terminated forcibly. It is well known that the solar atmosphere density is a function of altitude. The formula for density changes with height is:

$$
\rho=\rho_{0} e^{\left(-\frac{x}{115}\right)}\left(\rho_{0}=4 \times 10^{-7} \mathrm{~g} / \mathrm{cm}^{3}\right)[\mathbb{W}]
$$

In this formula, $\rho_{0}$ is a density of solar surface, its values is $4 \times 10^{-7} \mathrm{~g} / \mathrm{cm}^{3}, \mathrm{x}(\mathrm{x}=0,1,2 \ldots . .599)$ is a altitude above solar surface. However, Geant 4 can't changes the continuously in atmospheric density with height, so model should be simplified. We choose an attitude of $600 \mathrm{~km}$ from the surface of the sun to study and divided it into 600 regions. The density of each them has only density and information material density shown in Fig.2. We set energy threshold to $1 \mathrm{GeV}$, because the energy range has completely contain Fermi experimental spectrum observation range.

The second part is the cosmic rays setting. The proton is the most abundant component of cosmic rays. And accurate modeling of the contribution from other nuclei interactions is not very critical, therefor, whose contributions can be neglected. The proton is also the most abundant component of solar atmosphere, so we use $\mathrm{pp} \rightarrow \gamma$ interaction models, or, inelastic hadron interaction process [ए2]. Cosmic rays, or the protons, we deem, is isotropic without considering the magnetic field and other factors, this situations must be consideration into our discussion. Proton cosmic ray 


\section{density of solar atmophere}

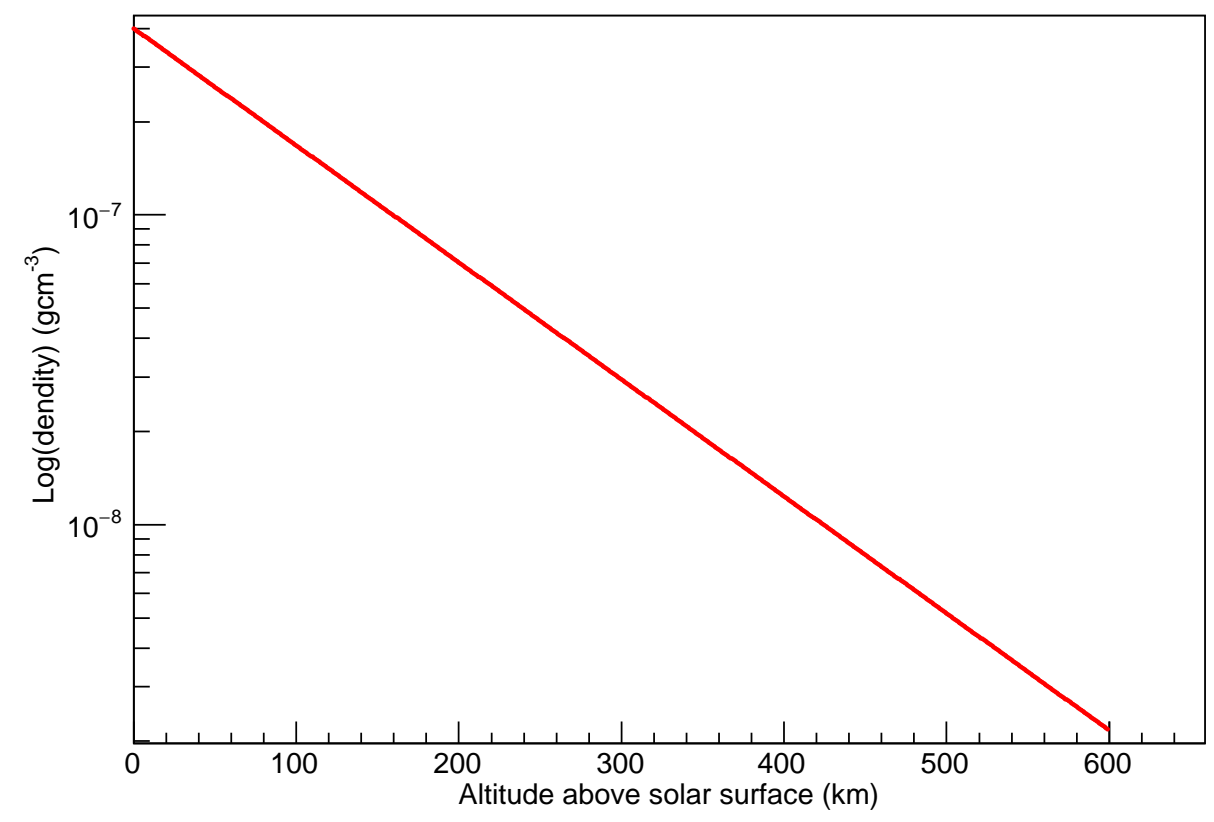

Figure 2: density of atmosphere above the solar surface

background flux is that:

$$
J_{B}(E)=8.3 \times 10^{-6} \times E^{-2.7}\left(\mathrm{~cm}^{-2} \mathrm{~s}^{-1} \mathrm{sr}^{-1} \mathrm{TeV}^{-1}\right)[\mathbb{[ 1 3}]
$$

The region, where above solar surface of $600 \mathrm{~km}$, are divided into 600 according to the height, the value of different parts of the density of solar atmosphere are given by formula 2.1 and spectral indices set to 2.7 .

\section{Data and analysis method}

The direction position and energy information of cosmic rays will be recorded as the raw data. The cosmic rays, or protons, enter into the solar atmosphere, and then interact with the proton among solar atmosphere, which produce solar gamma-ray, distribution of the direction position and energy information of which will be recorded either. We get the gamma-ray flux per unit area per unit time and normalized the result to the surface of the suns, we can get the flux of solar gamma-ray:

$$
\text { flu } u_{\text {gamma }}=\left(\frac{d N_{\text {gamma }}}{d E_{\text {gamma }}}\right) \times\left(\frac{1}{t_{\text {gamma }}}\right) \times\left(\frac{r^{2}}{R^{2}}\right)
$$

Where $r$ is the radius of the sun, the $\mathrm{R}$ is the average distance between the Earth and the Sun, we can get $t_{\text {gamma }}$ from proton of cosmic ray background flux, We don't discuss about them in detail any more in this paper. $d N_{\text {gamma }}$ is the number of gamma-ray, $d E_{\text {gamma }}$ is interval energy of gamma-ray, the we can get the $\left(\frac{d N_{\text {gamma }}}{d E_{\text {gamma }}}\right)$ from the raw data which we recorded from the detector we made, then multiplied by the $\left(\frac{1}{t_{\text {gamma }}}\right)$ and $\left(\frac{r^{2}}{R^{2}}\right)$. 


\section{Results and compares}

We record many kinds of the data and we show the information of Zenith angle distribution of solar gamma-ray, that the detector recorded in this paper, is shown in Fig.3.

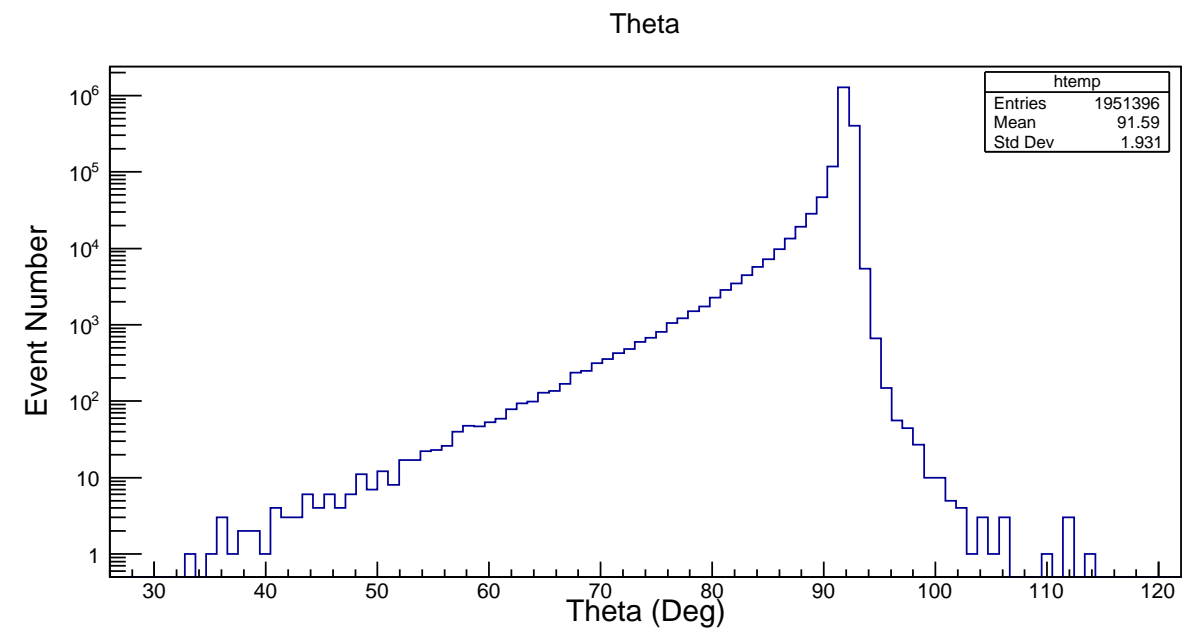

Figure 3: Zenith Angle distribution of solar gamma- ray

As shown in the Fig.1, the Cosmic rays, which enter into and interact with solar atmosphere, generate a bunch of particles. Zenith Angle of new particles are greater or less than Zenith Angle of Cosmic rays. The first situation is that Zenith Angle of new particles are greater than the cosmic rays, the higher the Zenith Angle the greater the absorbed probability $\left(\geq 90^{0}\right)$, if the particle enter into photosphere, as the same time, they are absorbed by atmosphere completely; in the opposite situation, new particles are scattered of the hundreds of times, Scattering Angle is proportional to the number of scattering $\left(0^{0} \sim 90^{\circ}\right)$, the two situations together contributed to the distribution of Zenith Angle. In order to improve the efficiency of the simulation, energy of flux of solar gamma-ray are divided into five, there are $5 \mathrm{GeV}-100 \mathrm{GeV}, 100 \mathrm{GeV}-1 \mathrm{TeV}, 1 \mathrm{TeV}-10 \mathrm{TeV}, 10 \mathrm{TeV}-$ $50 \mathrm{TeV}, 50 \mathrm{TeV}-100 \mathrm{TeV}$ respectively. We show information of the gamma-ray, which produced by hadronic interactions of cosmic rays with the solar atmosphere.

We show our results in condition of absence of magnetic field, our results provide a way to explore the source of the solar gamma-ray, or other source like it, mechanism of the new train of thought. It is well known to us that traditional calculation which are not suitable for scattering of the hundreds of times, Our approach can make up for the deficiency of the traditional calculation method. In order to verify the rationality of our results, we compare our results with the results of Zhou, et al (2017) [14]], which is shown in Fig.4.

In $50 \mathrm{GeV}-150 \mathrm{GeV}$ energy range, our results higher than the results of Zhou, et al (2017) [144] slightly. However, as a whole the results of our results with his fitting is very well. There are some reason of the difference we infer, such as density of detector the versions of Geant 4 between our and Zhou, et al (2017) [14] are different or the various parameters of detector have a reasonable range. In totally the error is in a reasonable range and the results is acceptable. The next stage, we will study what conduct the difference between us and provide the gamma-ray detector equipped with magnetic field environment . 


\section{flu of gamma-ray}

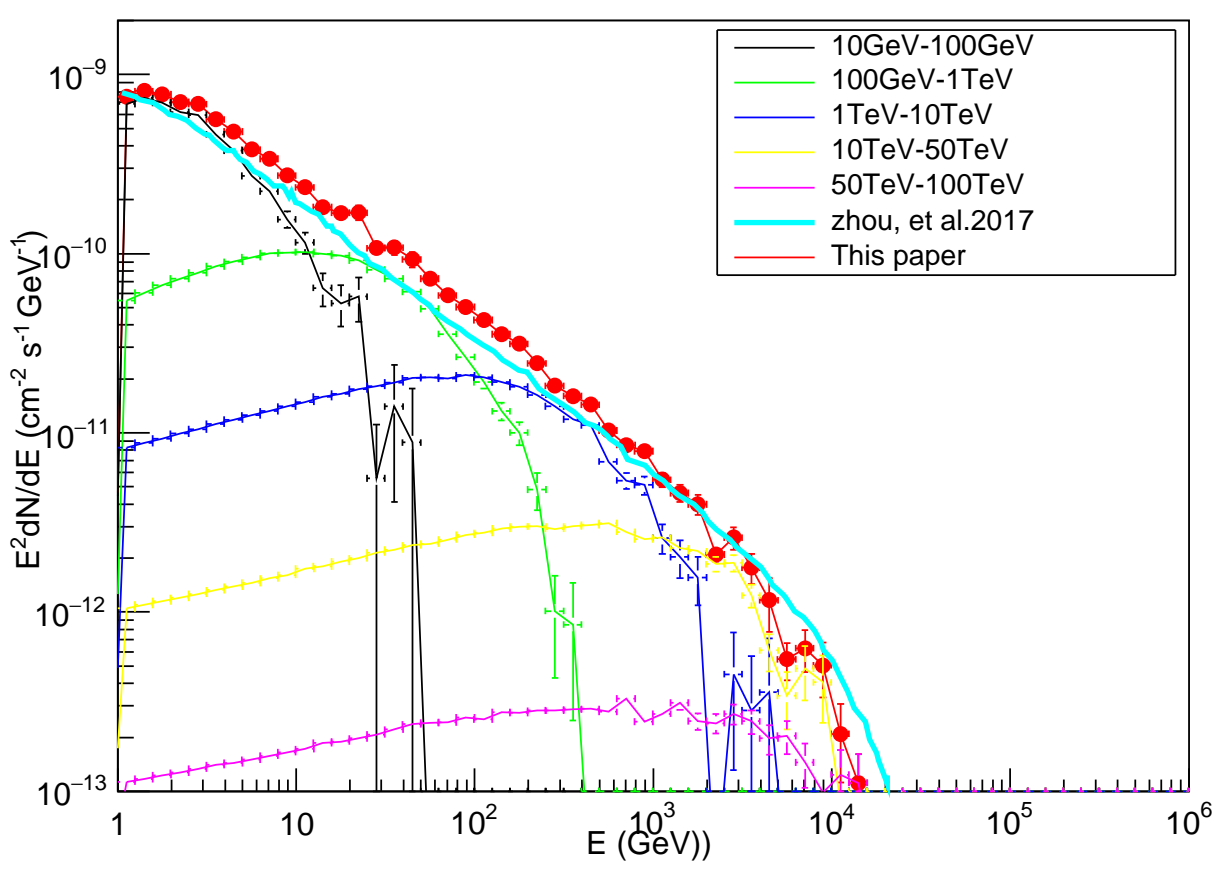

Figure 4: the fluxes of results between us and Zhou, et al 2017 [ㅁ]]

\section{CONCLUSIONS AND OUTLOOK}

Lots of factors are taken into account when we build the solar detector of gamma-ray. In order to make the detector simulate more effective, we divided the total energy range into five and set energy threshold to $1 \mathrm{GeV}$. We divided the solar atmosphere into 600 so that our simulation close to the truth as far as possible. The atmosphere of our solar detector is designed to be adjustable parameters, it is helpful to us to research the source of gamma-ray, which similar to the source of solar gamma-ray. We can study and simulate different source of gamma-ray by adjusting the parameters we set. Our work record the information of direction position and energy both proton and solar gamma-ray. Which can help us to a understand the scattering process easily. If the magnetic field is added to the detector successfully, we will study the magnetic field of the influence of the generation mechanism of cosmic rays in condition of complex field, and more factors will be considered in our model. Our study will become increasing reality.

\section{References}

[1] Ajello,M.,Albert,A.,Allafort,A.,et al.2014,APJ, 789, 20

[2] Ackermann,M.,Ajello,M.,Albert,A.,et al.2014,APJ,787, 15

[3] Atkins, R.,Benbow,W.,Berley,D.,et al.2004,Phys.Rev.D, 70,083516

[4] Bi,X.J.,Yin,P.F.,Yuan, Q, 2013,FrontiersofPhysics, 8, 794

[5] Abdo,A.A.,Ackermann,M.,Ajello,M., et al.2011,APJ,734, 116 
[6] Ng,K.C.Y.,Beacom,J.F.,Peter,A.H.G., Rott, C., 2016, Phys.Rev.D, 94.023004

[7] Dolan,J.F., andFazio, G.G., 1965, Geophys., 3, 319

[8] Peterson,L.E.,Schwartz,D.A.,Pelling,R.M., et al.1966,J.Geophys.Res., 71, 5778

[9] Seckel,D., Stanev, T., Gaisser,T.K.,APJ, 1991,382, 652

[10] Orlando,E., andStrong,A.W.2008,A\&A,480, 847

[11] D.Seckl,TodorStanev,ANDT.K.Gaisser.APJ.382 : 652 - 666, 1991

[12] http://geant4.cern.ch/support/proc ${ }_{m}$ od $_{c}$ atalog/physics $i$ ists/.

[13] JörgR.HÖrandel,.APJ.19(2003)193-220

[14] BeiZhou, KennyC.Y.Ng,JohnF.Beacom,AnnikaH.G.PeterarXiv : 1612.02420 